\title{
SEGURIDAD HOSPITALARIA Y DERECHOS HUMANOS
}

\author{
Dra. Ángeles de los Ríos Ruíz* \\ Dr. ANTONIO FUente DEL CAMPO**
}

\section{RESUMEN}

La seguridad sanitaria es, por naturaleza, colectiva y responsabilidad del sistema público de salud. Por lo que está relacionada con de la calidad de atención. Su significado está orientado a un balance positivo entre riesgos y beneficios, reduciendo los peligros asociados a la atención de salud. El tema de la seguridad es fundamental por sus implicaciones para la salud y la vida del ser humano. El derecho a la salud es un derecho humano consagrado en tratados internacionales y en las legislaciones nacionales. El cuidado de la salud ha llegado a ser una necesidad social y se ha reconocido la necesidad de adoptar programas de seguridad ante los riesgos y peligros existentes en el ambiente hospitalario, ya que de no hacerlo se pone en riesgo a todos los que colaboran y hacen uso del hospital, y también al medio ambiente.

\section{Palabras clave:}

Seguridad sanitaria, Derecho humano, Derecho a la salud, Salud, Calidad, atención médica.

\section{ABSTRACT}

Health security is, by nature, collective and the responsibility of the public health system. For what is related to quality of care. Its meaning is aimed at a positive balance between risks and benefits, reducing the dangers associated with health

* Profesora de Tiempo Completo “C” Definitiva de la Facultad de Derecho de la UNAM. Miembro del Sistema Nacional de Investigadores Nivel 1.

** Profesor titular del Posgrado en Cirugía Plástica, Estética y Reconstructiva, UNAM. Académico Emérito de la Academia Mexicana de Cirugía Plástica. Académico numerario de la Academia Nacional de Medicina de México. Miembro Honorario de la Asociación de Cirugía Plástica, Estética y Reconstructiva. Director de la clínica de la Cirugía Plástica Aqtuel. 
care. The issue of security is fundamental because of its implications for the health and life of the human being. The right to health is a human right enshrined in international treaties and national legislations. Health care has become a social necessity and the need to adopt safety programs in the face of the risks and dangers existing in the hospital environment has been recognized, since not doing so puts at risk all those who collaborate and do use of the hospital, and also the environment.

\section{Keywords:}

Health security, Human right, Right to health, Health, Quality, Medical care.

\section{EL DERECHO HUMANO A LA SALUD}

Los derechos humanos son derechos inherentes a todas las personas, sin distinción alguna de nacionalidad, lugar de residencia, sexo, origen nacional o étnico, color, religión, lengua, o cualquier otra condición. Además de los principios de universalidad y no discriminación, los derechos humanos están interrelacionados, son interdependientes e indivisibles; es decir, no se pueden respetar unos sí y otros no, aleatoriamente, porque unos influyen en el disfrute de otros.

\section{La salud como derecho humano}

La salud es mucho más que ausencia de enfermedad o tener acceso a la atención médica; es un derecho fundamental que toca todos los aspectos de la vida y por eso es tan importante entender la salud del modo más amplio posible. La Organización Mundial de la Salud estableció como definición de salud el estado de completo bienestar físico, mental y social.

A esta definición de salud se han ido incorporando otras dimensiones, como la capacidad de funcionar o la salud como un fenómeno continuo y dinámico a lo largo del tiempo, hasta llegar a instaurarse la idea de que la salud es un fenómeno multidimensional. Así pues, se constata que la noción de salud ha ido evolucionando a lo largo de la historia desde un enfoque médico-biológico hasta un concepto global e integral que incorpora el paradigma socio-ecológico (Frutos y Royo, 2006). ${ }^{1}$

El Derecho a la Salud se va desarrollando a través de las demandas sociales de los ciudadanos, conformando la definición actual de la salud, que es el estado de absoluto bienestar físico, mental y social, institucionalizada internacionalmente en 1946 con la Constitución de la Organización Mundial de la Salud (OMS). Más tarde, éste derecho encontrará un mayor desarrollo, dentro del área de los DESC. Es importante aclarar que, antes del desarrollo del Derecho a la Salud dentro de los organismos internacionales, el concepto se enfocaba en la idea de curar la enfermedad y los gobiernos hacían frente a los problemas de salud, desde la beneficencia pública. ${ }^{2}$

1 Prosalus y Cruz Roja Española (2014). “Comprendiendo el derecho humano a la salud”. Madrid, Edit. Advantia, pp. 114.

2 MONTIEL, L. (2004). "Derecho a la salud en México. Un análisis desde el debate teórico contemporáneo de la justicia sanitaria”. Revista IIDH, Vol. 40, pp. 291-313. 
Ahora, el Derecho a la Salud se considera como un derecho que integra no sólo la idea de curar la enfermedad, sino también de prevenirla, por lo que, el entorno físico y social del hombre adquiere una nueva relevancia dentro de este derecho. Esta nueva concepción de la salud implica una mayor protección del ser humano, así como un mayor compromiso administrativo y económico por parte de los Estados. Esta definición, caracterizada por su amplitud, pone de manifiesto la necesidad de delimitar el alcance y determinar las implicaciones del Derecho a la Salud.

Conocer los elementos que lo integran y sus características, resulta imprescindible para establecer un mecanismo de verificación y control de las obligaciones de los Estados.

El derecho a la salud debe entenderse como un derecho al disfrute de toda una gama de facilidades, bienes, servicios y condiciones necesarios para alcanzar el más alto nivel posible de salud. ${ }^{3}$

El derecho a la salud significa que los gobiernos deben crear las condiciones que permitan a todas las personas vivir lo más saludablemente posible. Esas condiciones incluyen la disponibilidad garantizada de servicios de salud, condiciones de trabajo saludable y seguro, vivienda adecuada y alimentos nutritivos. El derecho a la salud incluye el acceso a una atención sanitaria oportuna, aceptable, asequible y de calidad satisfactoria.

En este sentido, debe entenderse la salud es un estado de completo bienestar físico, mental y social, y no solamente la ausencia de afecciones o enfermedades.

El derecho a la salud está consagrado en tratados internacionales y regionales de derechos humanos y en las constituciones de países de todo el mundo y es un derecho humano reconocido por el derecho internacional de los derechos humanos.

El artículo 25 de la Declaración Universal de los Derechos Humanos consagra el derecho a la salud en los siguientes términos: "Toda persona tiene derecho a un nivel de vida adecuado que le asegure, así como a su familia, la salud y el bienestar, y en especial la alimentación, el vestido, la vivienda, la asistencia médica y los servicios sociales necesarios; tiene asimismo derecho a los seguros en caso de desempleo, enfermedad, invalidez, viudez, vejez u otros casos de pérdida de sus medios de subsistencia por circunstancias independientes de su voluntad."

En el Pacto Internacional de Derechos Económicos, Sociales y Culturales, se considera el instrumento fundamental para la protección del derecho a la salud, en este se reconoce el derecho de que toda persona disfrute del más alto nivel posible de salud física y mental. En el artículo 12 establece se establece que para que el Estado asegure la plena efectividad del derecho a la salud es necesario:

- La reducción de la mortalidad infantil y garantizar el sano desarrollo de los niños;

- El mejoramiento de la higiene del trabajo y del medio ambiente;

- La prevención y el tratamiento de las enfermedades epidémicas, endémicas, profesionales y de otra índole, y la lucha contra ellas;

3 Ídem. 
- La creación de condiciones que aseguren el acceso de todos a la atención de la salud.

En la Declaración Americana de los Derechos y Deberes del Hombre de 1948 se reconoce en el artículo XI, que toda persona tiene derecho a que su salud sea preservada por medidas sanitarias y sociales, relativas a la alimentación, el vestido, la vivienda y la asistencia médica, correspondientes al nivel que permitan los recursos públicos y de la comunidad.

La Convención sobre la Eliminación de todas las formas de Discriminación contra la Mujer (1979) dispone que los Estados Partes deberán adoptar las medidas necesarias para eliminar la discriminación contra las mujeres en la atención médica, a fin de asegurar el acceso a servicios de salud, inclusive los que se refieren a la planificación de la familia.

El Protocolo Adicional a la Convención Americana sobre Derechos Humanos en Materia de Derechos Económicos, Sociales y Culturales, mejor conocido como "Protocolo de San Salvador" (1988), reconoce que toda persona tiene derecho a la salud, como el disfrute del más alto nivel posible de bienestar físico, mental y social.

La Convención sobre los Derechos del Niño (1989) establece en su artículo 24 que los Estados Partes deben reconocer el derecho de las niñas y los niños al disfrute de la salud de más alto nivel, así como a los servicios para el tratamiento de enfermedades y rehabilitación.

El Comité de Derechos Económicos, Sociales y Culturales de las Naciones Unidas, en el año 2000 adoptó una observación general (número catorce del Comité de Derechos Económicos, Sociales y Culturales) sobre el derecho a la salud. En dicha observación general asevera que el derecho a la salud abarca no sólo la atención de salud oportuna, sino también, el acceso al agua limpia potable y a condiciones sanitarias adecuadas, el suministro adecuado de alimentos sanos, una nutrición adecuada, una vivienda adecuada, condiciones sanas en el trabajo y el medio ambiente, y acceso a la educación e información sobre cuestiones relacionadas con la salud, incluida la salud sexual y reproductiva.

El derecho a la salud, está estrictamente vinculado con el ejercicio de otros derechos humanos como: la vida, la dignidad humana, vivienda, alimentación, no discriminación, igualdad, acceso a la información, vida privada, entre otros. ${ }^{4}$

En este sentido, se puede observar que el derecho humano a la salud no se circunscribe solamente a la atención médica, sino que comprende una serie de factores socioeconómicos, mínimos necesarios, tales como: alimentación adecuada, agua limpia y potable, vivienda digna, condiciones de trabajo seguro, condiciones sanitarias adecuadas, entre otras.

Por tanto, la práctica médica debe llevarse a cabo desde las acciones singulares cotidianas de los profesionales y el equipo de salud con las personas, hasta la formulación e implementación de políticas públicas.

4 NOHELY BASTIDAS MATHEUS, "LA MALA PRÁCTICA MÉDICA Y LOS DERECHOS HUMANOS”, RAZÓN Y PALABRA Primera Revista Electrónica en América Latina Especializada en Comunicación, http://www.razonypalabra.org.mx/N/N81/M81/18_Bastidas_M81.pdf. 
El derecho a la salud comprende algunas libertades. Tales libertades incluyen el derecho a no ser sometido a tratamiento médico sin el propio consentimiento, por ejemplo experimentos e investigaciones médicas o esterilización forzada, y a no ser sometido a tortura $u$ otros tratos o penas crueles, inhumanos o degradantes. ${ }^{5}$

En este sentido, es obligación de los Estados Los Estados de proteger y promover los derechos humanos. Las obligaciones a este respecto están definidas y garantizadas por el derecho consuetudinario internacional y los tratados internacionales de derechos humanos, que imponen a los Estados que los han ratificado la obligación de hacer efectivos esos derechos.

Por lo cual resulta fundamental establecer mecanismos de rendición de cuentas para garantizar el respeto de las obligaciones que se derivan del derecho a la salud para los Estados. La vigilancia y la rendición de cuentas de los Estados tienen lugar a nivel nacional, regional e internacional e involucra a diversos agentes, a saber, el propio Estado, ONG, instituciones nacionales de derechos humanos $\mathrm{u}$ órganos creados en virtud de tratados internacionales. ${ }^{6}$

Ante el derecho a la protección de la salud surge la obligación de las instituciones médicas y de todo su personal a brindar una atención médica que colme dichos principios, por lo que si su actuación omitiere hacerlo éstos serían sujetos de una responsabilidad jurídica.

\section{Relación entre la salud y los derechos humanos}

Entre la salud y los derechos humanos existen vínculos complejos:

- La violación o la desatención de los derechos humanos pueden tener graves consecuencias para la salud;

- Las políticas y los programas sanitarios pueden promover los derechos humanos o violarlos, según la manera en que se formulen o se apliquen;

- La vulnerabilidad a la mala salud se puede reducir adoptando medidas para respetar, proteger y cumplir los derechos humanos. El contenido normativo de cada derecho se enuncia en su totalidad en los instrumentos de derechos humanos. A continuación, figuran algunos ejemplos de cómo está formulado en los instrumentos de derechos humanos el contenido normativo de algunos de los demás derechos humanos clave que guardan relación con la salud:

- Tortura: «Nadie será sometido a torturas ni a penas o tratos crueles, inhumanos o degradantes. En particular, nadie será sometido sin su libre consentimiento a experimentos médicos o científicos.»

- Violencia contra los niños: Violencia contra los niños: Se adoptarán «todas las medidas legislativas, administrativas, sociales y educativas apropiadas para proteger al niño contra toda forma de perjuicio o abuso

\footnotetext{
5 El derecho a la salud, Folleto informativo $N^{0} 31$, Oficina del Alto Comisionado de las Naciones Unidas para los Derechos Humanos, Ginebra 2008, p. 4.

6 Ibidem, p. 45.
} 
físico o mental, descuido o trato negligente, malos tratos o explotación, incluido el abuso sexual».

- Prácticas tradicionales nocivas: Prácticas tradicionales nocivas: Se adoptarán «medidas eficaces y apropiadas [...] para abolir las prácticas tradicionales que sean perjudiciales para la salud de los niños».

- Participación: El derecho a una participación «activa, libre y significativa».

- Información: «Libertad de buscar, recibir y difundir informaciones e ideas de toda índole.»

- Intimidad: «Nadie será objeto de injerencias arbitrarias o ilegales en su vida privada.»

- Progreso científico: El derecho de toda persona a gozar de los beneficios del progreso científico y de sus aplicaciones.

- Educación: El derecho a la educación, en particular a conocer los principios básicos de la salud y la nutrición de los niños, las ventajas de la lactancia materna, la higiene y el saneamiento ambiental y las medidas de prevención de accidentes, y a recibir apoyo para aplicar esos conocimientos.

- Alimentación y nutrición: «El derecho de toda persona a una alimentación adecuada y el derecho fundamental de toda persona a estar protegida contra el hambre».

- Nivel de vida: Toda persona tiene derecho a un nivel de vida adecuado, incluso alimentación, vestido y vivienda adecuados, y a la asistencia médica y los servicios sociales necesarios.

- Derecho a la seguridad social: El derecho de toda persona a la seguridad social, incluso al seguro social.

¿Qué se entiende por enfoque de la salud basado en los derechos humanos?

Por enfoque de la salud basado en los derechos humanos se entiende:

- Utilizar los derechos humanos como marco para el desarrollo sanitario.

- Evaluar las consecuencias que tiene cualquier política, programa o legislación sanitaria para los derechos humanos y adoptar medidas al respecto.

- Tener en cuenta los derechos humanos en la concepción, la aplicación, la supervisión y la evaluación de todos los tipos de políticas y programas (políticos, económicos y sociales, entre otros) que guarden relación con la salud.

Los principios fundamentales que habría que aplicar en esos procesos podrían ser los siguientes: ${ }^{7}$

$\checkmark$ Respetar la dignidad humana.

7 OMS (2002). “Veinticinco Preguntas y Respuestas Salud y derechos humanos”. Organización Mundial de la Salud. [Fecha de consulta: 02 de marzo de 2018] Recuperado de http://www.who.int/hhr/activities/Q\%26 AfinalversionSpanish.pdf 
$\checkmark$ Conceder atención a los grupos de la sociedad considerados más vulnerables. En otras palabras, reconocer y tener presentes las características de las personas a las que afectan las políticas, las estrategias y los programas sanitarios, esto es los niños y niñas, los adolescentes, las mujeres y los hombres; los pueblos indígenas y tribales; las minorías nacionales, étnicas, religiosas y lingüísticas; los desplazados internos; los refugiados; los inmigrantes y los migrantes; las personas de edad; las personas con discapacidad; los presos; los grupos de personas desfavorecidas desde el punto de vista económico o marginadas por algún otro motivo, y los grupos vulnerables.

$\checkmark$ Garantizar que los sistemas sanitarios se hagan accesibles a todos, especialmente a los sectores más vulnerables o marginados de la población, de hecho $\mathrm{y}$ de derecho, sin discriminación por ninguno de los motivos prohibidos.

$\checkmark$ Adoptar una perspectiva de género y reconocer que los factores biológicos y socioculturales influyen considerablemente en la salud de hombres y mujeres y que en las políticas y los programas es necesario tener presentes esas diferencias.

$\checkmark$ Garantizar la igualdad y la no discriminación, ya sea voluntaria o involuntaria, en la formulación y puesta en práctica de los programas de salud.

$\checkmark$ Desglosar los datos relativos a la salud para determinar si hay discriminación subyacente.

$\checkmark$ Garantizar la participación libre, fructífera y efectiva de los beneficiarios de las políticas o programas de desarrollo sanitario en los procesos de adopción de las decisiones que los afectan.

$\checkmark$ Promover y proteger el derecho a la educación y el derecho a buscar, recibir y difundir informaciones e ideas relativas a las cuestiones de salud. Ahora bien, el derecho a la información no debe menoscabar el derecho a la intimidad, lo que significa que debe darse un trato confidencial a los datos personales relativos a la salud.

$\checkmark$ Dejar que una política o un programa sanitario limite el ejercicio o el disfrute de un derecho únicamente como último recurso y no considerar que ello es legítimo salvo si se cumplen todas las disposiciones previstas en los Principios de Siracusa.

$\checkmark$ Confrontar las consecuencias para los derechos humanos de cualquier ley, política o programa sanitario con los objetivos de salud pública que se persiguen, y lograr que exista un equilibrio óptimo entre la obtención de resultados positivos desde el punto de vista de la salud pública y la promoción y protección de los derechos humanos.

$\checkmark$ Hacer referencias explícitas a las normas y reglas internacionales de derechos humanos para poner de relieve la forma en que los derechos humanos se aplican a una política, un programa o una ley sanitaria y la relación que existe entre ellos.

$\checkmark$ Perseguir como objetivo explícito fundamental de las actividades destinadas a mejorar la salud la realización del derecho al goce del grado máximo de salud que se pueda lograr. 
$\checkmark$ Enunciar las obligaciones concretas de los gobiernos de respetar, proteger y cumplir los derechos humanos.

$\checkmark$ Definir puntos de referencia e indicadores para supervisar la realización progresiva de los derechos en la esfera de la salud.

$\checkmark$ Aumentar la transparencia y exigir una gestión más responsable de las cuestiones de salud, como principio fundamental en todas las etapas del desarrollo de los programas.

$\checkmark$ Introducir salvaguardias para proteger de las principales amenazas a las minorías, los migrantes y otros grupos «impopulares» en los países, a fin de contrarrestar los desequilibrios de poder. Ejemplo de ello sería el establecimiento de mecanismos de recurso para los casos de violaciones de los derechos relacionados con la salud.

\section{SEGURIDAD SANITARIA}

Una obligación principal del gobierno con la población es proteger su vida y su salud. Este compromiso no es sólo ético, sino que debe contar con estructura institucional que lo respalde día con día. Ésta debe tener dos pilares básicos. Uno es un sistema de vigilancia e intervención epidemiológica con capacidad de detectar y enfrentar oportunamente una emergencia de salud. El otro es un sistema de protección contra riesgos sanitarios, por ejemplo, sustancias tóxicas en alimentos y bebidas, fármacos con efectos secundarios graves, publicidad engañosa, etcétera. ${ }^{8}$

La seguridad sanitaria es, por naturaleza, colectiva y responsabilidad del sistema público de salud. México cuenta formalmente con un sistema de vigilancia epidemiológica y una Comisión Federal de Protección contra Riesgos Sanitarios (Cofepris). Sin embargo, durante el año pasado ambos han probado ser más de papel que estructuras sólidas y actuantes. ${ }^{9}$

El tema de la seguridad es fundamental por sus implicaciones para la salud y la vida del ser humano. Toda organización debe asumir la gestión del riesgo de seguridad para lograr el compromiso de sus integrantes y prepararse para la identificación, prevención, reducción, eliminación, sustitución y mitigación de los factores que puedan desencadenar emergencias. Orientar la toma de decisiones para fortalecer las condiciones de la infraestructura, mitigar riesgos potenciales del ambiente, asumir el cumplimiento normativo de la gestión en la seguridad en los Hospitales; además de la aplicación de normas regulatorias antisísmicas en el diseño y la construcción de la infraestructura; aplicación de medidas sanitarias; conformación de brigadas de emergencias; Declaración de políticas de prevención y control de emergencias y desastres; aplicación de normas de construcción. Los anteriores aspectos deben ser vigilados por los entes territoriales, y declaración Universal de los Derechos Humanos. ${ }^{10}$

8 Laurell, A. C. (5 de febrero de 2010). "Seguridad sanitaria incierta". La Jornada. [Fecha de consulta: 29 de marzo de 2018] Recuperado de http://www.jornada.unam.mx/2010/02/05/ciencias/a03a1cie

9 Ídem.

10 GONZÁleZ, G.; PERTUZ, Y.; EXPÓSITO, M. Y. (2016). "Gestión de la seguridad hospitalaria en unidades de atención pediátrica”. En Revista Cubana de Enfermería, vol. 32(2), pp. 207-2017. 
La Calidad de Atención se ha convertido en los últimos años en un tema relevante en las agendas de Gobierno en materia de salud. Bajo las actuales exigencias en salud, las instituciones se han visto obligadas a incorporar este aspecto en la planificación de sus actividades, enfocadas principalmente a mantener un alto nivel en el continuo del cuidado de las personas. La seguridad es una de las dimensiones de la calidad de atención. Su significado está orientado a un balance positivo entre riesgos y beneficios, reduciendo los peligros asociados a la atención de salud. El cuidado de la salud ha llegado a ser una necesidad social y se ha reconocido la necesidad de adoptar programas de seguridad ante los riesgos y peligros existentes en el ambiente hospitalario, ya que de no hacerlo se pone en riesgo a todos los que colaboran y hacen uso del hospital, y también al medio ambiente. ${ }^{11}$

En nuestro país, la regulación de la práctica médica ha evolucionado con la participación de diversos actores: academias, colegios y asociaciones médicas; Consejo de Salubridad General, Secretaría de Salud, Comisión Nacional de Arbitraje Médico, Secretaría de Educación Pública y compañías aseguradoras; ¿pero regulación para qué?

El modelo de regulación sanitaria se ha concebido como una práctica de autoridad en relación al cumplimiento de normas y opera en un marco de rigidez que enfatiza sus acciones más hacia la búsqueda de omisiones que hacia la identificación de soluciones y fomento de buenas prácticas de seguridad sanitaria. ${ }^{12}$

La calidad, seguridad y efectividad de los servicios de atención médica son objetivos que conciernen a los gobiernos, a los profesionales de la salud y a la sociedad en su conjunto, y la gestión de riesgos en los servicios sanitarios es el conjunto de actividades destinadas a identificar, evaluar y reducir o eliminar el riesgo de que se produzca un suceso adverso que afecte:

- Las personas: pacientes, personal sanitario, directivos y demás trabajadores.

- Las instalaciones: edificios, equipos y dispositivos médicos, mobiliario, medio ambiente.

- Los recursos económicos: Inversiones, fondos de crecimiento y desarrollo, recursos de investigación.

- $\quad$ El prestigio y renombre de la institución y sus profesionales: satisfacción del personal, reputación, propiedad intelectual, relevancia, atracción de clientes.

La regulación sanitaria en México es un proceso permanente y dinámico ya que depende de la propia actividad humana, el desarrollo de nuevos productos y servicios; también de los avances tecnológicos y el descubrimiento de alternativas terapéuticas cada vez más complejas; además, la sociedad tiene cada día más

12 TRUjILlO, A. (2009). “Normatividad y regulación sanitaria en México”. Rev Mex Med Tran, vol. 2, Supl. 1, pp. S32-S34. 
conciencia de la calidad y de su seguridad; de esta manera se combina la gestión del riesgo y la gestión de la calidad en la elaboración del marco normativo para la regulación sanitaria. ${ }^{13}$

\section{EL SISTEMA DE SALUD EN MÉXICO}

El sistema de salud de México está compuesto por dos sectores: público y privado:

El sector público comprende a las instituciones de seguridad social tales como el Instituto Mexicano del Seguro Social (IMSS),Instituto de Seguridad y Servicios Sociales de los Trabajadores del Estado (ISSSTE),Petróleos Mexicanos (PEMEX),Secretaría de la Defensa Nacional (SEDENA), Secretaría de Marina (SEMAR) y otros], que prestan servicios a los trabajadores del sector formal de la economía, y a las instituciones que protegen o prestan servicios a la población sin seguridad social, dentro de las que se incluyen el Seguro Popular de Salud (SPS), la Secretaría de Salud (SSa),los Servicios Estatales de Salud (SESA) y el Programa IMSS-Oportunidades (IMSS-O).

El sector privado presta servicios a la población con capacidad de pago. El financiamiento de las instituciones de seguridad social proviene de tres fuentes:

a) Contribuciones gubernamentales.

b) Contribuciones del empleador (que en el caso del ISSSTE, PEMEX, SEDENA y SEMAR es el mismo gobierno)

c) Contribuciones de los empleados.

Estas instituciones prestan sus servicios en sus propias instalaciones y con su propio personal. Tanto la SSa como los SESA se financian con recursos del gobierno federal y los gobiernos estatales, además de una pequeña contribución que pagan los usuarios al recibir la atención (cuotas de recuperación). La población de responsabilidad de la SSa y de los SESA se atiende en las instalaciones de estas instituciones, que cuentan con su propio personal. El SPS se financia con recursos del gobierno federal, los gobiernos estatales y cuotas familiares, y compra servicios de salud para sus afiliados a la SSa y a los SESA (y en algunas ocasiones a proveedores privados). Finalmente, el sector privado se financia con los pagos que hacen los usuarios al momento de recibir la atención y con las primas de los seguros médicos privados, y ofrece servicios en consultorios, clínicas y hospitales privados. ${ }^{14}$

13 Ídem.

14 OCTAVIO GÓMEZ DANTÉS, et. al., 2Sistema de salud de México”, Salud Pública, número 2, vol. 53, México, 2011, p. 221. 


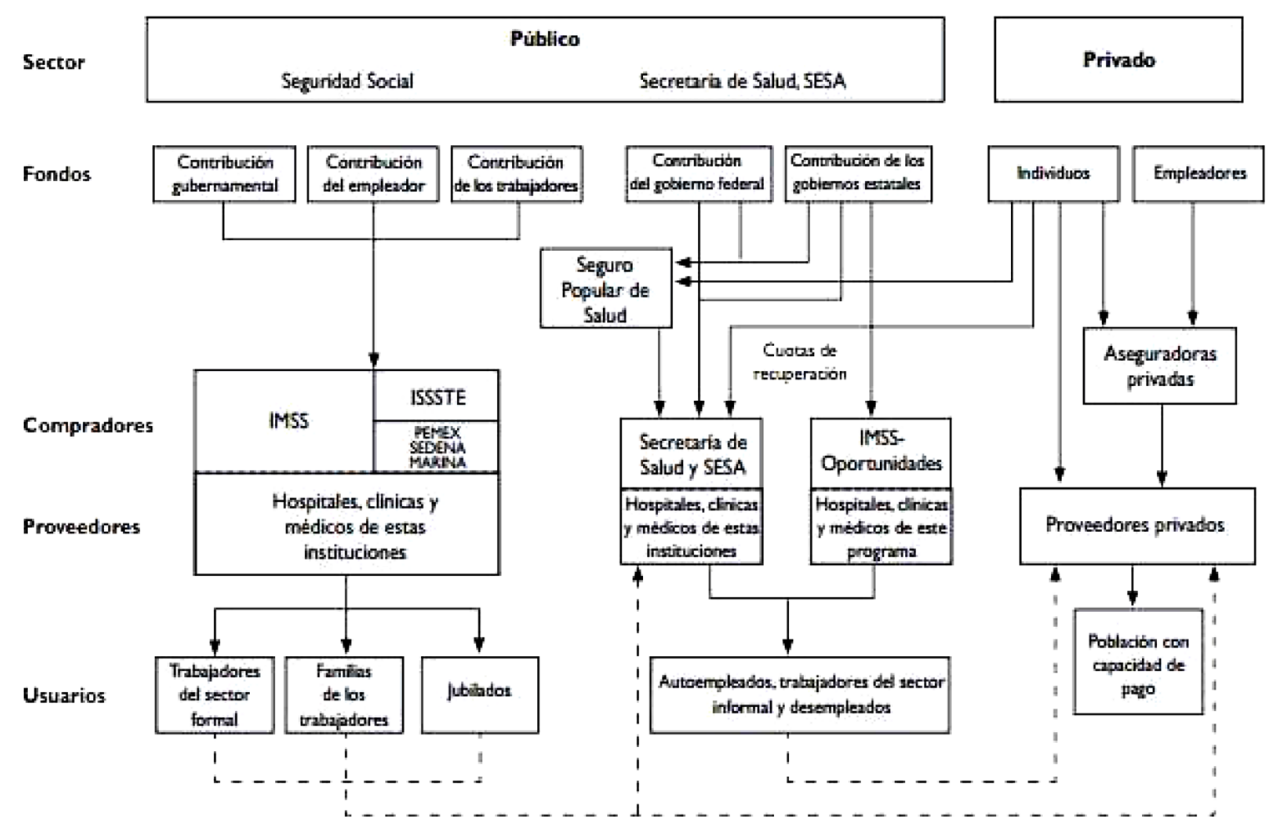

Sistema de salud en México

De acuerdo con el artículo $4^{\circ}$ de la Constitución Política de México, la protección de la salud es un derecho de todos los mexicanos. Sin embargo, garantizarlo es el gran problema que representa el sistema de salud en México. salud:

En el país hay tres distintos grupos de beneficiarios de las instituciones de

1. Los trabajadores asalariados, los jubilados y sus familias.

2. Los autoempleados, trabajadores del sector informal, desempleados y personas que se encuentran fuera del mercado de trabajo, y sus familias.

3. La población con capacidad de pago.

El sistema de la salud de México expresa rezagos importantes en tres indicadores centrales: equidad, calidad y cobertura financiera. Además, también se caracteriza porque se encuentra fuertemente segmentado, lo cual origina que el derecho a la salud no sea garantizado para toda la población. El gato público en salud ha estado concentrado en la población afiliada a la seguridad social, es decir, quienes acceden a los servicios de salud del IMSS o ISSSTE o PEMEX.

De acuerdo al Banco de México, el gasto total en salud es la suma del gasto público y privado en salud. Abarca la prestación de servicios de salud (preventivos y curativos), las actividades de planificación familiar, las actividades de 
nutrición y la asistencia de emergencia designadas para la salud, pero no incluye el suministro de agua y servicios sanitarios. ${ }^{15}$

El desempeño del Sistema de Salud en México durante los últimos años puede caracterizarse a través de dos procesos centrales y confluentes entre sí: la fragmentación y el deterioro de la calidad de sus servicios derivados de la reducción sistemática y progresiva del gasto público en la materia:

- La fragmentación del sistema de salud y minimización progresiva de los servicios otorgados a la población abierta.

- El decremento de la calidad en los servicios.

Este proceso de disminución del gasto público en salud tiene su contraparte en la implementación de mecanismos de privatización selectiva de los servicios de salud, debido a que la alternativa de dejar los servicios de salud en manos de particulares se enfocan primordialmente a aquellos servicios que por su costo intrínseco o por el poder adquisitivo de sus destinatarios, resultan más rentables. En contraposición, aquellos servicios poco rentables y que están destinados a la población de escasos recursos que en la mayoría de los casos forma parte del sector informal, siguen siendo considerados como una responsabilidad del Estado pero con una tendencia a ir restringiendo progresiva y marcadamente los recursos que se les asignan y los servicios que incluyen. ${ }^{16}$

Los indicadores del estado de salud de una nación son, en buena medida, reflejo de su nivel de desarrollo. De acuerdo a la OCDE, en el 2012, el gasto total en salud en México representó el 6.2\% de su PIB, este porcentaje se encuentra entre los más bajos de los países de la OCDE (sólo por encima de Estonia y Turquía) y muy por debajo del promedio de los países de la OCDE de 9.3\%. Como resultado de una gran expansión en la cobertura de salud para los pobres y los no asegurados que comenzó en el 2004, la participación pública en el financiamiento del cuidado de la salud en México se ha incrementado en alrededor de 10 puntos porcentuales para situarse en $50 \%$ en el 2012. Sin embargo, esta tasa se mantiene como una de las más bajas entre los países de la OCDE (donde el promedio es del 72\%), y alrededor de la mitad de todo el gasto en salud en México es pagado directamente por los pacientes. ${ }^{17}$

De acuerdo a información de Medigraphic de 2011,18 en México los prestadores de servicios de salud generan anualmente 187 millones de consultas generales, 41 millones de consultas de especialidad, 27 millones de consultas de urgencia, 1.5 millones de partos, 3.1 millones de intervenciones quirúrgicas y 4.5 millones de egresos hospitalarios. En el último trienio, más de 4.5 millones de consultas otorgadas.

15 Banco Mundial, http://datos.bancomundial.org/indicador/SH.XPD.TOTL.ZS

16 Alejandro Cerda García, México: "El derecho a la salud" en Helena Gardeazábal (coord.), Derecho a la salud. Situación en países de América Latina, ASOCIACIÓN LATINOAMERICANA DE MEDICINA SOCIAL, p. 152.

17 Estadísticas de la OCDE sobre la salud 2014 México en comparación, http://www.oecd.org/els/health-systems/ Briefing-Note-MEXICO-2014-in-Spanish.pdf

18 GERARDO RICARDO ZURITA NAVARRETE, op., cit., p. 42. 
En los primeros siete meses de 2009, las instituciones públicas del sector salud realizaron alrededor de 1.8 millones de intervenciones quirúrgicas. En ese mismo año, la ocupación hospitalaria registrada fue de $75 \%$.

El principal problema del Sistema Nacional de Salud en materia de calidad es la enorme heterogeneidad que existe a este respecto entre los principales prestadores de servicios.

En este tenor, es de mencionar el porcentaje de complicaciones de los partos vaginales en los hospitales de los SESA (Servicios de Salud) y de la Secretaría de Salud Federal $(0.48 \%)$ es 2.6 veces mayor que el porcentaje de los hospitales del IMSS (Instituto Mexicano del Seguro Social) (0.18\%).

El porcentaje de apéndices perforados en los hospitales de los SESA y de la Secretaría de Salud es mayor de $6 \%$ contra menos de $2 \%$ en los hospitales del IMSS y poco más de 3\% en las unidades hospitalarias del IMSS.

El porcentaje de readmisiones por colecistectomías abiertas en los hospitales menores de 60 camas del IMSS es de casi $2 \%$ contra menos de $1.5 \%$ en las unidades hospitalarias de la Secretaría de Salud.

El porcentaje de complicaciones de neumonías en pacientes de 60 años y más asciende a casi $27 \%$ en los hospitales del IMSS, a 14\% en los hospitales del ISSSTE y a menos de $23 \%$ en las unidades hospitalarias de los SESA y la Secretaría de Salud. A los problemas de calidad técnica habría que sumar los problemas de eficiencia.

El promedio de días estancia por hernioplastía inguinal en los hospitales de más de 120 camas del IMSS es de 1.5 días contra 2.6 días en los hospitales de los SESA y la Secretaría de Salud.

El promedio de días estancia para apendicectomías en hospitales menores de 60 camas del IMSS es 6.5 días contra 3 días en los hospitales de los SESA. E1 promedio de cirugías por quirófano en el IMSS es de casi 4 contra 2.2 en la Secretaría de Salud y 1.9 en PEMEX.

En los indicadores de calidad interpersonal también hay variaciones importantes. De acuerdo con la ENSA Nut 2006, el tiempo promedio de espera en consulta externa en el IMSS es de 90 minutos, contra menos de 30 minutos en las unidades del sector privado. El porcentaje de cirugías diferidas es de $20 \%$ en el ISSSTE, $18 \%$ en el IMSS, $18.2 \%$ en la Secretaría de Salud y $13 \%$ en el IMSS.

Los principales motivos de suspensión de cirugías son la falta de insumos, quirófanos y personal médico. Los usuarios de los servicios de la Secretaría de Salud son los que mejor califican la calidad de los alimentos y la limpieza de las instalaciones.

\section{EL DERECHO HUMANO A LA SALUD EN MÉXICO Y SEGURIDAD SANITARIA}

El concepto del derecho a la protección de la salud en México se ha ido transformando al paso de los años. En síntesis, este derecho se concebía inicialmente como una cuestión religiosa o de mera caridad; posteriormente, con base 
en el movimiento de Reforma, el Estado mexicano se asumió como el único responsable de su cumplimiento, pero bajo un aspecto meramente individualista; finalmente, la revolución de 1910 y las ideas progresistas del constituyente de 1917, transformaron esta concepción otorgando al derecho a la salud su carácter social, al señalar al Congreso de la Unión facultades en la materia. ${ }^{19}$

El derecho a la salud está consagrado en la Constitución Política de México en la fracción cuarta del artículo 4:

Toda persona tiene derecho a la protección de la salud. La Ley definirá las bases y modalidades para el acceso a los servicios de salud y establecerá la concurrencia de la Federación y las entidades federativas en materia de salubridad general, conforme a lo que dispone la fracción XVI del artículo 73 de esta Constitución. ${ }^{20}$

De la lectura de dicho párrafo se desprende que debe existir concurrencia entre la Federación y las Entidades Federativas para hacer efectivo el derecho a la salud. Lo cual responde no sólo a un sano federalismo, sino también a una necesidad real y un interés fundamental de los mexicanos por procurar que todas las instancias de gobierno intervengan en su concreción, ya que sin el concurso de ambas instancias (federal y estatal) la acción sanitaria sería del todo ineficaz.

Asimismo, debe decirse que una interpretación armónica de la Constitución permite inferir que los municipios también pueden ser partícipes de esta tarea, ya que el inciso i), fracción III del artículo 115 constitucional otorga la posibilidad de que los mismos se hagan cargo de los servicios públicos que determinen las legislaturas estatales. ${ }^{21}$

El derecho a la protección de la salud como auténtica garantía constitucional, además en el artículo $1^{\circ}$ reconoce en su fracción primera y segunda que el derecho a la salud es un derecho humano reconocido y consagrado por la Constitución:

En los Estados Unidos Mexicanos todas las personas gozarán de los derechos humanos reconocidos en esta Constitución y en los tratados internacionales de los que el Estado Mexicano sea parte, así como de las garantías para su protección, cuyo

19 EL DERECHO CONSTITUCIONAL A LA PROTECCIÓN DE LA SALUD. Su regulación constitucional y algunos precedentes relevantes del Poder Judicial de la Federación en México, PARTICIPACIÓN DE LA SEÑORA MINISTRA OLGA SÁNCHEZ CORDERO DE GARCÍA VILLEGAS, EN EL SIMPOSIO INTERNACIONAL "POR LA CALIDAD DE LOS SERVICIOS MÉDICOS Y LA MEJORÍA DE LA RELACIÓN MÉDICO PACIENTE", CELEBRADO EN EL AUDITORIO JAIME TORRES BODET DEL MUSEO NACIONAL DE ANTROPOLOGÍA E HISTORIA EN LA CIUDAD DE MÉXICO, EL 9 DE OCTUBRE DE 2000, www.scjn.gob.mx/conocelacorte/ministra/EL\%20DERECHO\%20CONSTITUCIONAL\%20A\%20 LA\%20PROTECCION\%20DE\%20LA\%20SALUD.pdf

20 Constitución Política de los Estados Unidos Mexicanos, http://www.dof.gob.mx/constitucion/marzo_2014 constitucion.pdf

21 EL DERECHO CONSTITUCIONAL A LA PROTECCIÓN DE LA SALUD. Su regulación constitucional y algunos precedentes relevantes del Poder Judicial de la Federación en México, PARTICIPACIÓN DE LA SEÑORA MINISTRA OLGA SÁNCHEZ CORDERO DE GARCÍA VILLEGAS, EN EL SIMPOSIO INTERNACIONAL "POR LA CALIDAD DE LOS SERVICIOS MÉDICOS Y LA MEJORÍA DE LA RELACIÓN MÉDICO PACIENTE”, CELEBRADO EN EL AUDITORIO JAIME TORRES BODET DEL MUSEO NACIONAL DE ANTROPOLOGÍA E HISTORIA EN LA CIUDAD DE MÉXICO, EL 9 DE OCTUBRE DE 2000, www.scjn.gob.mx/conocelacorte/ministra/EL\%20DERECHO\%20CONSTITUCIONAL\%20A\%20 LA\%20PROTECCION\%20DE\%20LA\%20SALUD.pdf 
ejercicio no podrá restringirse ni suspenderse, salvo en los casos y bajo las condiciones que esta Constitución establece. Las normas relativas a los derechos humanos se interpretarán de conformidad con esta Constitución y con los tratados internacionales de la materia favoreciendo en todo tiempo a las personas la protección más amplia. ${ }^{22}$

El derecho a la protección de la salud además de encontrar su contenido específico en el artículo 4to Constitucional, también las disposiciones legislativas secundarias, reglamenta y amplían los contenidos del derecho a la protección de la salud constitucionalmente consagrado.

La Ley General de Salud en su artículo 2do establece las finalidades del derecho a la protección a la salud:

El derecho a la protección de la salud, tiene las siguientes finalidades:

I. El bienestar físico y mental del hombre para contribuir al ejercicio pleno de sus capacidades;

II. La prolongación y el mejoramiento de la calidad de la vida humana;

III. La protección y el acrecentamiento de los valores que coadyuven a la creación, conservación y disfrute de condiciones de salud que contribuyan al desarrollo social;

IV. La extensión de actitudes solidarias y responsables de la población en la preservación, conservación, mejoramiento y restauración de la salud;

V. El disfrute de servicios de salud y de asistencia social que satisfagan eficaz y oportunamente las necesidades de la población;

VI. El conocimiento para el adecuado aprovechamiento y utilización de los servicios de salud, y;

VII. El desarrollo de la enseñanza y la investigación científica y tecnológica para la salud. ${ }^{23}$

En la Ley General de Salud se encuentran los siguientes criterios mínimos del derecho a la salud:

a) En el artículo 35 establece que los servicios públicos de salud deben ser prestados bajo los criterios de universalidad y gratuidad. ${ }^{24}$

b) El artículo 25 que se debe garantizar la extensión cuantitativa y cualitativa de los servicios, preferentemente a grupos vulnerables..$^{25}$

c) El artículo 36 indica que las cuotas de recuperación que en su caso se recauden deberán tomar en cuenta el costo de los servicios y las condiciones socioeconómicas de los usuarios, fundándose en principios de solidaridad social y eximiendo del cobro a los usuarios que carezcan de recursos para cubrirlas o que se encuentren las zonas de menor desarrollo económico y social del país. ${ }^{26}$

22 CONSTITUCIÓN POLÍTICA DE LOS ESTADOS UNIDOS MEXICANOS, Última reforma publicada DOF 07-07-2014, http://www.diputados.gob.mx/LeyesBiblio/htm/1.htm

23 LEY GENERAL DE SALUD, http://www.salud.gob.mx/unidades/cdi/legis/lgs/LEY_GENERAL_DE_SALUD.pdf

26 Ídem. 
d) El artículo 6 establece que el Sistema Nacional de Salud deberá prestar servicios a toda la población y mejorar la calidad de los mismos, dando prioridad a las acciones preventivas. ${ }^{27}$

e) El artículo $77 \mathrm{Bis} 1$ establece que todos los mexicanos tienen derecho a ser incorporados al Sistema de Protección Social en Salud de conformidad con el artículo cuarto de la Constitución Política de los Estados Unidos Mexicanos, sin importar su condición social. Además, que la protección social en salud es un mecanismo por el cual el Estado garantizará el acceso efectivo, oportuno, de calidad, sin desembolso al momento de utilización y sin discriminación a los servicios médico-quirúrgicos, farmacéuticos y hospitalarios que satisfagan de manera integral las necesidades de salud. ${ }^{28}$

En este sentido, la legislación secundaria del derecho a la salud, tiene un carácter orgánico que no configura verdaderas prerrogativas de los ciudadanos exigibles al Estado. Al respecto existen algunos criterios emitidos por el Poder Judicial de la Federación. ${ }^{29}$

El derecho a la protección de la salud ha sido interpretado por el Poder Judicial de la Federación como un derecho fundamental que encuentra su consagración a nivel constitucional y su contenido específico en la regulación secundaria. Los derechos sociales son normas, y como tales deben tener efectos vinculatorios para los sujetos obligados, entre los que se encuentran, por supuesto, los poderes públicos.

De tal manera que el derecho a la salud como un derecho humano no puede entenderse sin la garantía de seguridad sanitaria. La cual engloba la prevención y los excelentes cuidados asistenciales, y esta acción es directamente proporcional a la participación y compromiso con los políticos en la distribución de los recursos. ${ }^{30}$

El derecho a la protección de la salud tiene, entre otras finalidades, la de garantizar el disfrute de servicios de salud y de asistencia social que satisfagan las necesidades de la población, y que por servicios de salud se entienden las acciones dirigidas a proteger, promover y restaurar la salud de la persona y de la colectividad, lo cual es compatible con varios instrumentos internacionales de derechos humanos.

\footnotetext{
7 Ídem.

28 Ídem.
}

29 PARTICIPACIÓN DE LA SEÑORA MINISTRA OLGA SÁNCHEZ CORDERO DE GARCÍA VILLEGAS, EN EL SIMPOSIO INTERNACIONAL "POR LA CALIDAD DE LOS SERVICIOS MÉDICOS Y LA MEJORÍA DE LA RELACIÓN MÉDICO PACIENTE”, CELEBRADO EN EL AUDITORIO JAIME TORRES BODET DEL MUSEO NACIONAL DE ANTROPOLOGÍA E HISTORIA EN LA CIUDAD DE MÉXICO, EL 9 DE OCTUBRE DE 2000, https://www.google.com.mx/url?sa=t\&rct=j\&q=\&esrc=s\&source=web\&cd= $3 \&$ cad $=$ rja\&uact $=8 \&$ ved $=0 \mathrm{CCYQFjAC} \&$ url $=$ https $\% 3 \mathrm{~A} \% 2 \mathrm{~F} \% 2 \mathrm{Fwww} \cdot \mathrm{scj}$.gob.mx $\% 2 \mathrm{~F}$ conocelacorte $\% 2 \mathrm{Fm}$ inistra\%2FEL \%2520DERECHO \%2520CONSTITUCIONAL\%2520A\%2520LA\%2520PROTECCION\%25 20DE\%2520LA\%2520SALUD.pdf\&ei=uTT3VK3sJpb_yQTX_oGoBw\&usg=AFQjCNFun2ALJ0aftGDkC 29c_VGgXjsV8Q\&sig2=e4OXRQzk_-D0A2lnfx5BKg\&bvm=-bv.87519884,d.aWw

30 LOY̌, M. \& DÍAZ, H. (2009). "Hospitales en México". En Revista Cir Ciruj, Volumen 77, pp. 497-504. 


\section{CONCLUSIONES}

- El Estado Mexicano debe garantizar el derecho a la salud en la mayor medida posible de acuerdo a los recursos disponibles, incluso cuando éstos sean escasos, pero deben procurar cumplir sus obligaciones de respeto, protección y realización del derecho humano a la salud. Para ello, es necesario el fortalecimiento de las competencias de los profesionales de la salud con relación al conocimiento no sólo de su materia y la pericia para aplicarla, sino también del conocimiento de la aplicación de los instrumentos internacionales de derechos humanos, principalmente en el contexto de la eficiencia y calidad de la atención a los pacientes y a sus derechos humanos.

- El derecho humano a la salud no puede entenderse sin la garantía de seguridad sanitaria. La cual engloba la prevención y los excelentes cuidados asistenciales. El marco jurídico del derecho humano a la salud es la base para: a) unificar estrategias que mejoren la salud de los grupos sociales más pobres y excluidos; b) mejorar la equidad en salud; c) aclarar la rendición de cuentas y las responsabilidades de los sistemas de salud, y d) evaluar el cumplimiento de las recomendaciones al Estado Mexicano.

- El derecho a la salud forma parte del derecho al desarrollo humano y tiene una doble dimensión: es un derecho del constitucionalismo social mexicano para la protección de la salud, y un derecho de solidaridad, porque actualmente, la salud es vista como un problema internacional.

- El principal problema del sistema de salud en México en materia de calidad es la enorme heterogeneidad que existe entre los principales prestadores de servicios. Por lo que el mayor reto es buscar alternativas para fortalecer su integración, de tal manera que se garantice un paquete común de beneficios a todas las personas, se reduzcan los altos costos de transacción inherentes a un sistema segmentado y se logre finalmente el ejercicio universal e igualitario del derecho a la protección de la salud.

- Una buena práctica médica obedece a centros de salud bien equipados, suficiente personal médico para atender a los pacientes, excelente formación médica de los profesionales de la salud y salarios bien pagados a estos. Sin embargo, en México, el problema fundamental radica en el aumento de población que acude al sector público para atención médica, lo cual provoca que ante una numerosa cantidad de consultas, cuya corta duración, limita la posibilidad de entablar una relación cordial médico-paciente. La falta de tiempo para la atención médica de cada paciente en forma particularizada puede hacer incurrir al médico en un actuar imprudente, negligente o erróneo.

\section{FUENTES DE INFORMACIÓN}

Banco Mundial, http://datos.bancomundial.org/indicador/SH.XPD.TOTL.ZS CERDA GARCÍA Alejandro, México: "El derecho a la salud" en Helena Gardeazábal (coord.), Derecho a la salud. Situación en países de América Latina, ASOCIACIÓN LATINOAMERICANA DE MEDICINA SOCIAL. 
El derecho a la salud, Folleto informativo $\mathrm{N}^{\mathrm{o}} 31$, Oficina del Alto Comisionado de las Naciones Unidas para los Derechos Humanos, Ginebra 2008.

Estadísticas de la OCDE sobre la salud 2014 México en comparación, http://www. oecd.org/els/health-systems/Briefing-Note-MEXICO-2014-in-Spanish.pdf

GÓMEZ DANTÉS Octavio, Sistema de salud de México", Salud Pública, número 2, vol. 53, pp. México, 2011.

GONZÁlEZ, G.; PERTUZ, y., EXPÓSITO, M. Y. (2016). “Gestión de la seguridad hospitalaria en unidades de atención pediátrica”. En Revista Cubana de Enfermería, vol. 32(2), pp. 207-2017.

Laurell, A. C. (5 de febrero de 2010). "Seguridad sanitaria incierta". La Jornada. [Fecha de consulta: 29 de marzo de 2018] Recuperado de http://www.jornada.unam.mx/2010/02/05/ciencias/a03alcie

LEY GENERAL DE SALUD, http://www.salud.gob.mx/unidades/cdi/legis/lgs/ LEY_GENERAL_DE_SALUD.pdf

MONTIEL, L. (2004). "Derecho a la salud en México. Un análisis desde el debate teórico contemporáneo de la justicia sanitaria”. Revista IIDH, Vol. 40, pp. 291-313.

NOHELY BASTIDAS MATHEUS, "LA MALA PRÁCTICA MÉDICA Y LOS DERECHOS HUMANOS", RAZÓN Y PALABRA, Primera Revista Electrónica en América Latina Especializada en Comunicación, http://www.razonypalabra.org.mx/N/N81/M81/18_Bastidas_M81.pdf.

OMS (2002). "Veinticinco Preguntas y Respuestas Salud y derechos humanos". Organización Mundial de la Salud. [Fecha de consulta: 02 de marzo de 2018] Recuperado de http://www.who.int/hhr/activities/Q\%26AfinalversionSpanish. pdf

Prosalus y Cruz Roja Española (2014). "Comprendiendo el derecho humano a la salud". Madrid, Edit. Advantia.

TRUJILLO, A. (2009). "Normatividad y regulación sanitaria en México". Rev Mex Med Tran, Vol. 2, Supl. 1, pp S32-S34. 